RUNNING HEAD: Instructed Evaluative Conditioning

\title{
Examining the Contributions of Memory-Dependent and Memory-Independent Components to Evaluative Conditioning via Instructions
}

\author{
Mandy Hütter \\ Eberhard Karls Universität Tübingen \\ $\&$ \\ Jan De Houwer \\ Ghent University \\ in press, Journal of Experimental Social Psychology
}

\begin{abstract}
Author Notes
Mandy Hütter, Fachbereich Psychologie, Eberhard Karls Universität Tübingen, Germany; Jan De Houwer, Department of Experimental-Clinical and Health Psychology, Ghent University, Belgium.

The preparation of this article was supported by an Emmy-Noether grant from the Deutsche Forschungsgemeinschaft (HU 1978/4-1) awarded to the first author. Jan De Houwer is supported by Methusalem Grant BOF16/MET_V/002 of Ghent University and an Interuniversity
\end{abstract}


Attraction Poles grant of the Belgian Science Policy Office (P7/33). We thank Olivier Corneille and Christoph Klauer for helpful comments on a previous version of this manuscript.

Correspondence concerning this article should be addressed to Mandy Hütter at the Fachbereich Psychologie, Eberhard Karls Universität Tübingen, Schleichstr. 4, 72076 Tübingen, Germany. Electronic mail may be sent to mandy.huetter@uni-tuebingen.de. 


\begin{abstract}
We investigated whether instructions have the potential to generate memory-independent attitude acquisition as indexed by a stochastic model of evaluative conditioning that distinguishes between memory-dependent and memory-independent learning. For that purpose, we instructed participants about pairings of conditioned and unconditioned stimuli without having participants experience them. We obtained a significant contribution of memory-independent learning that depended on whether instructions emphasized the importance of memorization at learning or the importance of feelings at either learning or retrieval. Our findings call for caution when interpreting the memory-independent contribution as an indicator of association formation on the one hand and unaware learning on the other hand. Our research demonstrates the need to clearly distinguish between processes operating at encoding and processes operating at retrieval in empirical and theoretical research on evaluative conditioning.
\end{abstract}

Keywords: evaluative conditioning, instructed learning, awareness, automaticity, dualprocess models 
Psychologists have long recognized that human behavior is strongly shaped by preferences (Zajonc, 1968). It is thus of importance to understand how preferences develop. Previous research suggests that many preferences are learned rather than innate (Rozin \& Millman, 1987). A paradigm assigned a key role in this research is evaluative conditioning (EC). In EC studies, a conditioned stimulus (CS) is paired with an unconditioned stimulus (US) that typically carries valence (i.e., is positive or negative). As an effect of that pairing procedure, the CS typically acquires the valence of the US. The EC effect has been demonstrated with a wide variety of stimuli and pairing procedures (Hofmann, De Houwer, Perugini, Baeyens, \& Crombez, 2010). The nature of the processes underlying this effect has been debated intensely and approached with different correlational and experimental methodologies (e.g., Hofmann et al., 2010; Sweldens, Corneille, \& Yzerbyt, 2014).

Interestingly, it has been argued that EC is only found when there is evidence for awareness of the CS-US contingencies (Pleyers, Corneille, Luminet, \& Yzerbyt, 2007; Stahl, Unkelbach, \& Corneille, 2009) and when the learning situation allows for the encoding of the pairings in explicit memory (Dedonder, Corneille, Bertinchamps, \& Yzerbyt, 2013; Stahl, Haaf, \& Corneille, 2016). This claim is controversial, because it is at odds with the idea that learning in general can proceed implicitly (e.g., Reber, 1993), that is, in the absence of conscious knowledge about the environmental regularities that drive learned changes in behavior. Claims that EC depends on contingency awareness have also featured heavily in the debate about the processes that mediate EC. More specifically, it has been taken as evidence for the role of propositional processes in EC and as evidence against association formation models (e.g., Hofmann et al., 2010). Whereas association formation models attribute EC to the (relatively) automatic formation of CS-US associations in memory, propositional models postulate that EC depends on the nonautomatic formation of conscious propositional beliefs about the relation between the CS and US. 
In contrast to simple associations, propositions can specify the way in which CS and US are related (e.g., CS causes US, CS predicts US, CS is equivalent to the US, etc.). Hence, from the perspective of propositional models, EC is not a primitive mechanism that creates preferences in a bottom-up manner, but a complex cognitive phenomenon that has much in common with other complex phenomena such as problem solving and persuasion (De Houwer, 2009; De Houwer \& Hughes, 2016).

However, the evidence for the role of awareness of the CS-US contingencies - and thus the evidence for propositional models - might have been overestimated. As argued by Hütter, Sweldens, Stahl, Unkelbach, and Klauer (2012), participants might use the conditioned valence of a CS as a valid cue for inferring the valence of a US (e.g., "I like the CS, so probably it was paired with a positive US”). Such an affect-as-information strategy would result in a strong relation between EC and indices of CS-US contingency awareness even though EC might have occurred in the absence of contingency awareness. Hütter et al. (2012) thus used multinomial processing tree (MPT) modelling (Batchelder \& Riefer, 1999; Hütter \& Klauer, 2016) to separate awareness from conditioned responses. In a memory task administered after conditioning, participants were asked to report for each CS whether it was paired with pleasant or unpleasant US pictures. Additionally, participants were instructed to report their evaluation of the CS when they did not remember the US valence. The MPT model underlying the estimation of these processes is depicted in Figure 1. In the inclusion condition, participants remember US valence with the probability $m$. If they lack memory for the pairings (with the probability $1-m$ ), the reported evaluation of the CSs is in line with US valence if the conditioning procedure generated representations that influence liking in the absence of memory. The probability of that process is estimated by the parameter $a$. If participants lack memory for the pairings and the learning phase did not generate evaluations in the absence of memory, participants simply guess. The parameter 
$r$ represents the probability of giving a “pleasant” response in the absence of any learning. As both memory-dependent and memory-independent learning lead to the same responses in the inclusion condition, those processes cannot be dissociated on the basis of performance in this condition alone. Therefore, Hütter et al. also implemented an exclusion condition, in which they asked participants to reverse the responses based on their evaluations. That is, when participants remembered the valence of the US pictures with which a CS was paired, they simply had to report the valence of the US pictures (i.e., "pleasant” or "unpleasant” as in the inclusion condition). However, when they did not remember the valence of the US pictures, they were asked to respond in a way that is opposite to their evaluation of the CS picture (i.e., respond “pleasant” if they disliked the picture and response "unpleasant” if they liked the picture). In this exclusion condition, memory-dependent and memory-independent effects are in opposition (e.g., remembering that a CS was paired with a positive stimulus would result in selecting the “pleasant” response whereas feeling good about the CS in the absence of memory about the CSUS pairing would result in selecting the “unpleasant” response). ${ }^{1}$ By taking into account performance in both the inclusion and exclusion condition, three parameters can be estimated unambiguously (see Figure 1). Most importantly, the $a$-parameter is designed to capture evaluations in the absence of memory for the pairings. The estimation of this parameter should thereby evade the problem of affect-as-information that potentially contributes to memory performance in conventional memory tasks. The $m$-parameter should thus also be relatively free of affect-as-information processes and reflect actual memory of the CS-US pairings.

The results of the MPT modeling by Hütter et al. showed that next to the encoding of stimulus relations in explicit memory (i.e., a significant $m$-parameter), there was also a contribution of memory-independent learning to EC (i.e., a significant $a$-parameter). Based on 
these findings, Hütter et al. concluded that EC can be unaware and thus that associative processes contribute to EC.

However, there are two problems with this conclusion. First, as was the case with most studies on the role of contingency awareness, Hütter et al. (2012) assessed memory for the CSUS pairings after the presentation of the CS-US pairs rather than during the CS-US pairings. As pairings might be forgotten during the time between the learning and the test phase (also see Lovibond \& Shanks, 2002), the MPT findings might tell us more about the relation between EC and memory at the time of retrieval rather than between EC and contingency awareness (see Gawronski \& Walther, 2012, for a detailed discussion of this problem). Second, it is possible in principle that propositional knowledge also influences behavior in the absence of memory for the stimulus relations. Whereas propositional models postulate that EC depends on the non-automatic formation of conscious propositional beliefs about the CS-US relation, they allow for the possibility that these beliefs can be retrieved and thus influence subsequent behavior in an automatic manner (De Houwer, 2014). Hence, observing significant memory-independent learning in MPT studies does not necessarily argue against the idea that a conscious proposition about the CS-US relation has to be formed before EC can arise.

In the present research, we set out to examine the validity of the second objection to the conclusions of Hütter et al. (2012), namely that MPT analyses do not provide a pure index of associative processes, because propositions also can have memory-independent effects, that is, influence preferences in the absence of explicit memory for the pairings. If this argument is correct, one should be able to find evidence for memory-independent learning even when participants are merely instructed about the CS-US pairings, but do not actually experience those pairings. De Houwer (2006; also see Gast \& De Houwer, 2012) already demonstrated that mere instructions about CS-US pairings can change the liking of the CS. Proponents of propositional 
models of EC interpret this finding as support for those models, because they assume that instructions about CS-US contingencies result in propositions about those contingencies, but do not allow for the formation of associations. Hence, if the instructions lead to changes in liking, this could be due only to the formation of propositional knowledge. If an effect of instructions on the $a$-parameter in a MPT analyses can be observed, this would thus strengthen the conclusion that the $a$-parameter does not provide a pure index of associative processes. Note, however, that such a finding would not necessarily provide unique support for propositional models. As pointed out by others (e.g. Gawronski \& Bodenhausen, 2006), it might well be that instructions do allow for the formation of associations in memory and that effects of instructions on liking or MPT parameters are mediated by associative processes. Nevertheless, finding an impact of instructions on the $a$-parameter would show that it is at least plausible that propositions influence that parameter and would thus strengthen calls for caution when interpreting MPT parameters. Hence, for the first time, we used the MPT model developed by Hütter and colleagues (2012) to test whether instructions can also have memory-independent effects, as would become apparent in a significant $a$-parameter.

As a second goal, we will investigate the impact of instructions present at the retrieval stage on the presumed indicators of processes operating during learning. Because the MPT model relies on a memory measure that is applied only after learning and because the model is based on self-report data, it is important to understand the role of retrieval and response processes that operate during the memory measure on which MPT relies. Any effects on the MPT parameters observed may be due to processes operating during learning, retrieval, or both. For instance, MPT estimates could depend on the subjective criteria that participants use to decide whether they remember or do not remember the CS-US pairings. As the MPT model draws a distinction between stimulus evaluations and memory for the pairings, we vary in the present experiments 
whether instructions emphasize memory or feelings, thereby inducing strict or lenient criteria of responding based on memory or feelings. Given that participants in this task are left to decide on the basis of which process (i.e., memory or feelings) they respond, it is possible that small variations in the instructions influence the results that may be drawn about learning.

Emphasizing and thereby varying response thresholds regarding memory also helps evaluating the MPT approach more generally. One assumption of MPT models is that memorydependent and memory-independent processes are independent of another (e.g., Jacoby, 1991). An alternative model for the conceptualization as independent (dual) processes is that the parameters do not represent two qualitatively different indicators of memory-dependent and memory-independent learning, but memory of high and low strength, respectively, as in a signaldetection framework (Swets, 1996; Wixted, 2007). Specifically, such an account assumes that memory strength is a continuum along which participants set thresholds, using high strength stimuli for memory-based responding and low strength stimuli for attitude-based responding (Wixted, 2007). The dual-process account can be tested against the signal-detection account empirically. Specifically, if the two processes are independent of each other, manipulations of memory thresholds should only have an effect on memory-dependent learning, but not on memory-independent learning. Only a single study informs this question so far (Hütter et al., 2012, Exp. 3). In that study, not the memory threshold was manipulated, but the quality of memory itself. Specifically, memory-dependency of evaluative learning was assessed immediately after the conditioning phase or only after 24 hours had passed. The estimate of memory-independent learning was not affected by this manipulation, while memory-dependent learning was significantly diminished. 


\section{Overview of Experiments}

We conducted three experiments on the role of memory-independent EC in instructed procedures. All of these studies relied on an MPT model that distinguishes three processes in a retrieval task: memory-dependent learning, memory-independent learning, and guessing (Hütter et al., 2012). The first experiment compared the parameter structure for a typical instructed (De Houwer, 2006) and a typical pairing procedure (Hütter et al., 2012). More specifically, we tested whether only the typical pairing procedure (i.e., CS-US pairings are actually presented) would produce a significant $a$-parameter. Experiment 2 served to investigate the role of memorization instructions that were confounded with the nature of the procedure (pairings or instructions) in Experiment 1. Finally, Experiment 3 investigated the role of a focus on feelings both during learning and during the retrieval task to explore a potential explanation for the effects of memorization instructions revealed by Experiment 2.

\section{Data collection, sample sizes, and reporting of results}

We generally aimed at collecting 20 participants (or 480 observations) per condition and stopped data collection at the end of the day that goal was reached (Exp. 1) or collected more data when the lab routines allowed (Exp. 2, 3). That is, because the experiments constituted the first experiment in multi-experiment sessions that lasted up to an hour, we increased sample size in accordance with other studies that required a larger sample size. The continuation of data collection was never contingent on preliminary data analysis.

The MPT model analyses were based on 1104 (Exp. 1), 1368 (Exp. 2), and 1968 (Exp. 3) observations, that is, 24 CSs per participant. As a rule of thumb for sufficient power, Klauer, Stahl, and Voss (2011) suggested that not more than 10\% of the expected category counts should be below five. With the present sample size and parameter estimates, the smallest expected category counts amount to 43 (Experiment 1), 90 (Experiment 2), and 55 (Experiment 3). 
We report all measures, manipulations, and exclusions in the respective sections of each experiment.

\section{Experiment 1}

\section{Method}

Participants. We collected data from 48 students at the University of Heidelberg. Two participants were excluded because they gave the same response in $88 \%$ and $100 \%$ of the trials in the memory task, respectively. The analyses thus included the data of 46 participants (28 females, 18 males; $\left.M_{\text {age }}=24.54, S D_{\text {age }}=6.78\right)$.

Design. The experiment employed a 2 (US valence: positive vs. negative) $\times 2$ (time of measurement: preratings vs. postratings) $\times 2$ (type of procedure: pairings vs. instructions) $\times 2$ (instructions at retrieval test: inclusion vs. exclusion) mixed design with repeated measures on the first two factors.

Materials and Procedure. All the instructions, presentations, and measures were implemented in a customized $\mathrm{C}++$ computer program. Participants first rated the valence of the 120 black-and-white pictures of human faces (60 males, 60 females). Evaluative ratings were assessed on a continuous scale with the endpoints "very unpleasant" and "very pleasant" that was translated into a 200-point scale by the computer program. For each participant, the 24 most neutrally rated stimuli were selected as CSs. Twelve CSs were randomly assigned to positive US valence and 12 CSs were assigned to negative US valence.

As USs, we used 50 pleasant and 50 unpleasant pictures from the International Affective Picture System (IAPS; Lang, Bradley, \& Cuthbert, 1999). Pleasant pictures were more positive than unpleasant pictures, $t(98)=60.77, p<.001$. They did not differ in terms of arousal, $t(98)=-$ $.02, p=.98$, but differed in potency, $t(98)=19.66, p<.001$. That is, unpleasant pictures were more dominant than pleasant pictures. 
In the pairing procedure, participants watched six pairings of each CS with different USs. CS-US pairs were presented for $2000 \mathrm{~ms}$ each and separated by an inter-trial interval of $100 \mathrm{~ms}$. Participants watched a total of 144 pairings.

In the instructed procedure, participants received a description of the conditioning phase that allegedly would follow at a later stage of the experiment without seeing the actual pairings: In an upcoming perception phase of this study, the faces that you just rated will be presented together with pleasant or unpleasant pictures. The pleasant pictures depict, for example, babies, pets, or flowers. The unpleasant pictures show, for example, garbage, injuries, or obviously dangerous animals. A given face will be presented six times together with different either pleasant or unpleasant pictures.

You will now see two consecutive screens. On one screen, you will see the faces that will be presented with pleasant pictures during the perception phase. On the other, you will see the faces that will be presented with unpleasant pictures. Each screen will be presented for 2.5 minutes. The transition between screens will proceed automatically. It is very important that you remember which face will be presented with which type of pictures. You will definitely need this information to finish the task successfully.

Participants were then presented with two picture sets consisting of the CSs to be paired positively on the one hand, and the negative CSs on the other hand. The order of the presentation of the sets was randomized. The picture sets were headed "These faces will be presented with PLEASANT (UNPLEASANT) pictures during the perception phase.” Each of the picture sets were presented for 151 seconds, which is exactly 50 percent of the total duration of the pairing procedure. 
The conditioning phase was followed by a memory task tailored to the MPT model proposed by Hütter and colleagues (2012). For each of the 24 CSs, participants reported whether it had been paired with pleasant or unpleasant pictures by clicking on one of the two category boxes labeled “pleasant” and "unpleasant.” Participants in the inclusion condition were instructed to respond in accordance with their memory. If they did not remember those pairings, they were instructed to report their evaluation of the face using the same response boxes. In the exclusion condition, participants were also instructed to report their memory of the pairings and the evaluation of the face when they lacked memory for the pairings. However, they were required to reverse their responses based on their attitude toward the CS when they did not remember the pairings.

In order to verify that participants comprehended the memory task instructions, practice trials were included after the instructions were given. The practice trials comprised verbal scenarios that systematically varied the factors valence of the paired pictures by valence of the face. For example, participants were told that a face was shown with pleasant pictures and that at the same time they evaluated the face positively. They were asked to indicate their response in cases where (a) they remembered the pairings and (b) they did not remember the pairings. For each scenario, participants had to indicate the appropriate response for cases (a) and (b). In the case of false responses, the instructions were repeated and another practice round started. The instructions and practice trials were conducted up to three times. If an error was made in the final practice repetition, participants were excluded from analysis.

Finally, participants rated once more all CSs in random order on the evaluative rating scale used for the pre-ratings.

No other measures were collected and no additional manipulations were employed. 


\section{Results}

Evaluative Ratings. Ratings were submitted to a 2 (US valence) $\times 2$ (time of measurement) $\times 2$ (type of procedure) mixed General Linear Model (GLM). The EC effect (US valence by time of measurement) was significant, $F(1,44)=11.69, p<.001, \eta_{\mathrm{p}}{ }^{2}=.21$, and not moderated by the type of procedure, $F(1,44)=0.03, p=.87, \eta_{\mathrm{p}}{ }^{2}=.001$. The main effect of US valence was also significant, $F(1,44)=12.61, p<.001, \eta_{\mathrm{p}}{ }^{2}=.22$, irrespective of type of procedure, $F(1,44)=0.02, p=.90, \eta_{\mathrm{p}}{ }^{2}=.00$. Time of measurement did not exert an effect on ratings, $F(1,44)=0.43, p=.52, \eta_{\mathrm{p}}{ }^{2}=.01$, independent of type of procedure, $F(1,44)=1.35, p=$ $.25, \eta_{\mathrm{p}}^{2}=.03$. Mean evaluative ratings are presented in Figure 2 .

MPT Model. We fitted a model with one $m$-, one $a$-, and one $r$-parameter per type of procedure, that fit the data well, $G^{2}(2)=1.84, p=.40$. Subsequently, we observed the change in model fit when setting the parameters equal across conditions. The $m$-parameter was smaller in the experienced condition, $m=.32$, 95\% confidence interval (CI) $[.24, .40]$, than in the instructed condition, $m=.51$, 95\% CI $[.43,58], \Delta G^{2}(1)=11.98, p<.001$. The $a$-parameter was larger in the experienced condition, $a=.11,95 \%$ CI $[.00, .22]$, than in the instructed condition, $a=.00$, $95 \%$ CI $[.00, .15], \Delta G^{2}(1)=3.10, p=.07 .^{2}$ In the experienced condition, the $a$-parameter's estimate differed significantly from zero, $\Delta G^{2}(1)=3.82, p<.05$, but not in the instructed condition, $\Delta G^{2}(1)=0.00, p=.99$. The $r$-parameter indicated a tendency to use the "unpleasant" response option in the experienced procedure, $r=.37,95 \%$ CI $[.31, .44]$, which was not present in the instructed procedure, $r=.51,95 \%$ CI $[.43, .58], \Delta G^{2}(1)=4.90, p<.05$. The parameter estimates are depicted in Figure 3.

\section{Discussion}

Results showed that the strength of evaluative learning as indexed by ratings was not influenced by whether participants were merely instructed about the pairings or actually 
perceived them. However, the MPT model indicates that different processes gave rise to these learning effects in the two conditions. While memory-independent learning was present in the experienced procedure thereby replicating previous findings (Hütter \& Sweldens 2013; Hütter et al., 2012), the EC effect was due only to memory-dependent processes in the instructed procedure. This finding argues against the idea that propositional knowledge can have effects in the absence of conscious memory of CS-US pairings and thus seems to support the conclusion of Hütter et al. (2012) that a significant $a$-parameter in MPT studies indicates the operation of associative processes.

However, the two types of procedures differed not only in whether participants watched actual pairings. Specifically, the instructed procedure but not the experienced procedure included an instruction to memorize the US valence. We included this instruction, because it was used in previous studies on EC via instructions (e.g., De Houwer, 2006; Gast \& De Houwer, 2012). An alternative explanation for the present findings arises from this difference. That is, because the MPT model depends on participants' explicit responses and because it is left to participants to judge whether they remember the valence of the pairings, memory instructions could influence responding at test. That is, if instructions emphasize memory, participants could feel inclined to indicate that they remember the CS-US relation based only on their feelings towards the CS. As a result, explicit memory would be overestimated and memory-independent learning underestimated. If instructions have the power to shift responses between the $m$ - and $a$ parameters, the conclusions that are drawn from the MPT model should be treated with caution.

\section{Experiment 2}

If the alternative explanation discussed for Experiment 1 influenced the results, then instructed procedures could lead to a significant $a$-parameter when memory-instructions are 
omitted. In the next experiment, we thus used an instructed procedure and manipulated the presence of memory instructions.

\section{Method}

Participants. Sixty University of Tübingen students took part in this study. We excluded three participants from analysis, who still made errors in the third and final practice round. The resulting sample consisted of 47 women and 10 men of 18 to 41 years of age $(M=23.39, S D=$ 5.20).

Design. The experiment employed a 2 (US valence: positive vs. negative, withinparticipants) $\times 2$ (time of measurement: pre-rating vs. post-rating) $\times 2$ (memory-instructions: control vs. memory emphasized) $\times 2$ (instructions at retrieval test: inclusion vs. exclusion) mixed design with repeated measures on the first two factors.

Materials and Procedure. The memory instructions were identical to the instructions given in Experiment 1. In the control condition, we simply told participants that they could familiarize themselves with the faces and prepare for the upcoming phase. The two conditions thus differed only with regard to the fact that the following sentence was not part of the instructions in the control condition: "It is very important that you remember which face will be presented with which type of pictures. You will definitely need this information to finish the task successfully.”

No other measures were collected and no additional manipulations were employed.

\section{Results}

Evaluative Ratings. A GLM revealed a significant EC effect, $F(1,55)=27.87, p<.001$, $\eta_{\mathrm{p}}{ }^{2}=.34$, which was also apparent in a main effect of US valence, $F(1,55)=13.00, p<.001, \eta_{\mathrm{p}}{ }^{2}$ $=.19$. Time of measurement did not exert a main effect on the ratings, $F(1,55)=0.33, p=.57$, $\eta_{\mathrm{p}}{ }^{2}=.01$. The EC effect was dependent on instruction condition, $F(1,55)=4.65, p<.05, \eta_{\mathrm{p}}{ }^{2}=$ 
.08. The EC effect was larger in the control condition, $F(1,28)=20.43, p<.001, \eta_{\mathrm{p}}{ }^{2}=.42$, than in the condition that instructed participants to memorize the pairings, $F(1,27)=7.88, p<.01, \eta_{\mathrm{p}}{ }^{2}$ $=.23$. These EC effects are illustrated in Figure 4.

MPT Model. We fitted an initial model with one $m$-, one $a$-, and one $r$-parameter per condition. This model offers an adequate description of the data obtained, $G^{2}(2)=4.89, p=.09$. The $m$-parameter was larger in the control condition, $m=.71,95 \%$ CI $[.65, .76]$, than in the memorization condition, $m=.61,95 \% C I[.55, .67], \Delta G^{2}(1)=5.78, p<.05$. In the control condition, we obtained an estimate of the $a$-parameter, $a=.43,95 \% C I[.27, .59]$, that was significantly larger than zero, $\Delta G^{2}(1)=22.17, p<.001$, and significantly larger than its estimate in the memorization condition, $a=.00,95 \%$ CI [.00, .15], $\Delta G^{2}(1)=18.43, p<.001$. The $r$ parameters could be set equal across the control condition, $r=.61$, 95\% CI [.47, .76], and the memorization condition, $r=.56,95 \% C I[.48, .63], \Delta G^{2}(1)=0.48 p=.49$. The resulting estimate was $r=.57,95 \% C I[.50, .63]$. The final model with five parameters, which is depicted in Figure 5, fit the data well, $G^{2}(3)=5.37, p=.15$.

\section{Discussion}

The EC effect on the evaluative ratings was present in both the control and the memorization conditions. However, this effect was significantly larger when participants were not instructed to memorize the pairings. Surprisingly, the $m$-parameter was smaller in the memorization condition than in the control condition. Furthermore, the $a$-parameter was also smaller in the memorization condition than in the control condition. Thus, we did not find evidence for the use of affect-as-information as memory (if that was the case, the $m$-parameter should have increased in the memorization condition as the $a$-parameter decreased). These findings do indicate, however, that memorization instructions raise the thresholds for the report of both memory and evaluation. At the process level, stronger $m$ - and $a$-parameters indicate that 
participants guess less often in the control condition than in the memorization condition. The proportion of guessing can be obtained by multiplying the converse probabilities of $m$ and $a$. In the memorization condition, the proportion of guessing amounts to $39 \%$, in the control condition to $17 \%$. In general, the parameter estimates in the two conditions mirror the magnitude of the EC effects. ${ }^{3}$

Of primary importance for the goals of our paper, however, is the fact that we did observe a significant $a$-parameter in the control condition, even though only instructions were given about CS-US contingencies and CS-US pairs were not actually presented. The absence of memoryindependent learning in the first experiment is thus not due to the absence of the CS-US pairings. Instead, it seems to be the case that adding a sentence that emphasizes the importance of memorization of CS-US pairs reduces the MPT estimate of memory-independent learning. In sum, the present experiment demonstrates that memory-independent learning is also possible in instructed procedures. The present experiment does not, however, shed light on the mechanisms by which memorization instructions decrease MPT estimates of memory-dependent and memoryindependent learning. The next experiment was designed to shed light on this question.

\section{Experiment 3}

Experiment 3 addressed a possible mechanism by which the memorization instructions reduced MPT estimates of both memory-dependent and memory-independent learning in the previous experiment. One possibility is that memorization instructions reduced the focus on feelings during learning and/or testing. This might have interfered with the processing of the valence of the stimuli and thus reduced EC and/or reliance of feelings during test (e.g., Gast \& Rothermund, 2011). To examine this possibility in Experiment 3, we compared a condition with memorization instructions only to conditions in which memorization instructions were combined with instructions that emphasized the importance of feelings. If memorization instructions have 
their detrimental impact because they reduce the focus on valence, then these effects could be compensated by the additional feeling instructions. The two conditions with feeling instructions differed with regard to the time at which the instructions were administered. In one condition, the feeling instructions were given prior to the conditioning phase. This allowed for an impact of the feeling instructions both during learning and during test. In a second condition, the feelings instructions were presented only at test, thus allowing for an impact at test only. A comparison of these two conditions thus allowed us to examine when feeling instructions have an effect.

\section{Method}

Participants. Eighty-nine students at University of Tübingen took part in the present study. We excluded seven participants, who still made errors in the third and final practice round of the memory task. Analyses included the data of 64 female and 18 male students who were between 18 and 31 years of age $(M=22.71, S D=3.00)$.

Design. The experiment employed a 2 (US valence: positive vs. negative) $\times 2$ (time of measurement: pre-rating vs. post-rating) $\times 3$ (feeling instructions: control vs. feelings during learning emphasized vs. feelings during memory test emphasized) $\times 2$ (instructions at retrieval test: inclusion vs. exclusion) mixed design with repeated measures on the first two factors.

Materials and Procedure. The control condition replicated the memorization condition of the previous experiment, which is also almost identical to the instructed condition in Experiment 1. Hence, the instructions in this condition included the sentence that emphasized the importance of remembering the instructions about the CS-US contingencies. In two additional conditions, we also included the memorization instructions but added instructions that emphasized feelings either at learning or at test. The feelings-at-acquisition condition was identical to the control condition with two sentences added that emphasized the importance of affective responses: “At 
the same time, you should pay attention to your feelings towards the pictures. Previous research has already shown that feeling-based learning is of great importance.”

The feelings-at-retrieval condition was identical to the control condition except that the instructions for the memory test contained three additional sentences (here for the exclusion condition): "It is very important that you also follow the instructions carefully when you do not remember the images that a face was to be presented with. If you cannot remember what was about to happen, it is very important that you listen to your feelings toward the face and select the response that is opposite to those feelings. This task might seem strange, but prior research has shown that such responses do lead to meaningful results, provided that the judges respond on the basis of their feelings.”

No other measures were collected and no additional manipulations were employed.

\section{Results}

Evaluative Ratings. A GLM indicated a significant EC effect, $F(1,79)=13.78, p<.001$, $\eta_{\mathrm{p}}{ }^{2}=0.15$, and a main effect of US valence, $F(1,79)=12.49, p<.001, \eta_{\mathrm{p}}{ }^{2}=0.14$. Time of measurement did not exert a main effect, $F(1,79)=0.77, p=.38, \eta_{\mathrm{p}}{ }^{2}=0.01$. None of these effects was moderated by feelings instructions, all $F$ 's $<0.22$, smallest $p=.80$. The EC effects are presented in Figure 6.

MPT Model. The current data required the estimation of separate $a$-parameters for positively and negatively paired CSs (as models that set the two equal as in the previous experiments did not exhibit sufficient fit), a model with zero degrees of freedom. We then successively set parameters equal across conditions. The $m$-parameters in the two conditions that emphasized feelings did not differ from another (feelings-at-acquisition: $m=.63,95 \%$ CI [.56, .69]; feelings-at-retrieval: $m=.59,95 \% C I[.53, .65]), \Delta G^{2}(1)=0.80, p=.37$. The $m$-parameter 
in the control condition differed from the others, $m=.28,95 \% C I[.20, .35]), \Delta G^{2}(1)=60.18, p<$ .001.

The estimate of the $a$-parameter for positively paired CSs was zero in both the control condition, $a^{+}=.00,95 \% C I[.00, .14]$, and the feelings-at-acquisition condition, $a^{+}=.00,95 \%$ $C I[.00, .22]$. The estimates did not differ from another, $\Delta G^{2}(1)=0.00, p=1$. The $a$-parameter in the feelings-at-retrieval condition, $a+=.49,95 \% C I[.30, .68]$ was significantly larger than the estimate of the other two conditions, $\Delta G^{2}(1)=16.32, p<.001$.

For the negatively paired CSs, the feelings-at-retrieval condition, $a-=.55$, $95 \% C I[.35$, .74], did not differ from the feelings-at-acquisition condition, $a-=.44,95 \% C I$ [.23, .64], $\Delta G^{2}(1)$ $=0.70, p=.40$, but differed significantly from the control condition, $a-=.31,95 \% C I[.17, .45]$, $\Delta G^{2}(1)=4.12, p<.05$. The feelings-at-acquisition condition, however, was not significantly different from the control condition, $\Delta G^{2}(1)=0.97, p=.32$.

The $r$-parameter's estimate in the feelings-at-acquisition condition, $r=.50$, 95\% CI [.40, $.60]$, did not differ from the control condition, $r=.53$, 95\% $C I[.47, .59], \Delta G^{2}(1)=0.32, p=.57$, while the feelings-at-retrieval condition did, $r=.38,95 \% C I[.23, .53], \Delta G^{2}(1)=3.48, p=.06$, indicating a tendency to respond “unpleasant.” The two conditions that emphasized feelings did not differ significantly from another, $\Delta G^{2}(1)=1.81, p=.18$. The final model with eight parameters constituted a satisfactory account of the data, $G^{2}(4)=2.49, p=.65$, and is illustrated in Figure 7.

\section{Discussion}

EC effects on the evaluative ratings were obtained irrespective of instructions. Most important for the present purpose, we again found significant $a$-parameters despite the absence of actual CS-US pairings. This confirms that instructions about CS-US contingencies are sufficient to obtain memory-independent learning as indexed by MPT analyses. 
We also observed that the MPT parameters depended on the exact instructions that participants received during acquisition and test. First, the fact that the $a$-parameter did not differ from zero in the control condition again suggests that memorization instructions interfere with the effect of instructions on memory-independent learning as estimated by MPT. Second, if anything, estimates of the $a$-parameter were larger in the feelings-at-retrieval condition than in the feelings-at-acquisition conditions. If feelings instructions exert their impact mainly during acquisition (e.g., by increasing the evaluative processing of the USs), one would have expected the largest $a$-parameters in the feelings-at-acquisition condition. Hence, our findings suggest that feeling instructions have their impact mainly at the stage of the memory test.

The third experiment also helps us to refute alternative explanations for the MPT parameters. First, the fact that the $m$-parameter was not larger in the control condition than in the emphasis-on-feelings conditions indicates again that affect-as-information did not compromise the estimate of the $m$-parameter. Second, the fact that increases (decreases) in the $a$-parameter were not accompanied by decreases (increases) in the $m$-parameter contradicts the notion that the $a$-parameter is comprised of low-confidence memory rather than memory-independent evaluations. The increase in both the $m$ - and the $a$-parameters rather means that participants show less guessing in these conditions than in the control condition. In the control condition, participants guess in about $60 \%(1-m \times 1-a)$ of the cases. In the feelings-at-retrieval condition, this proportion is reduced to about $20 \%$. Thus, both the $a$-parameter and the $m$-parameter are influenced by lower response thresholds. In summary, with a focus on feelings at retrieval participants utilize also weaker products of (memory-dependent and memory-independent) learning in this self-report measure. 


\section{General Discussion}

In three experiments, we assessed the effects of instructions on the parameters of an MPT model tailored to the measurement of memory-dependency of evaluative learning. Our results suggest that memory-independent EC as indexed by MPT parameters is not a function of instructed versus experienced procedures per se, but a matter of memorization instructions that decrease the utilization of memory and evaluations at retrieval. In line with this idea, we observed that a focus on feelings during retrieval enhances both the estimates of memory-dependent and memory-independent learning and reduces the probability of random guessing. These findings are in line with the idea that the $a$-parameter does not provide a pure index of associative processes, because propositions that are based on instructions can also generate memoryindependent effects, that is, influence preferences in the absence of explicit memory of the CSUS pairings. Hence, our results lend credence to the possibility that also in studies with actual pairings, propositions can produce a significant $a$-parameter. Our results do not provide conclusive evidence for propositional models (De Houwer, 2009; Mitchell et al., 2009), because instructions might also lead to associations (Gawronski \& Bodenhausen, 2006), but they do show that the propositional account of the $a$-parameter is at least plausible and that one should therefore be careful in interpreting the $a$-parameter as a proxy for associative processes.

In the present studies, we did not implement a concurrent measure of contingency awareness, in part because of the many difficulties associated with that type of measure (e.g., Shanks \& St. John, 1994). Hence, we cannot make definite statements about the actual levels of contingency awareness during encoding. However, because participants in the instruction groups were actually told which CSs would appear with positive pictures and which CSs would appear with negative pictures, it is almost certain that participants’ awareness of the CS-US contingencies was high. It is interesting to note that we obtained a significant $a$-parameter in the 
instructions group despite the (presumably) high levels of contingency awareness. This finding thus provides an illustration of the general point that EC in the absence of contingency memory during test (which is, in the case of the present studies, indicated by a significant $a$-parameter) should not be equated with EC in the absence of contingency awareness during acquisition. This cautionary note applies not only to the interpretation of the $a$-parameter but to all measures of contingency memory (Gawronski \& Walther, 2012). In order to make more definite conclusions about the role of contingency awareness during learning, the MPT approach has to be combined with experimental manipulations of encoding conditions (e.g., Dedonder et al., 2013; Mierop, Hütter, \& Corneille, in press; Stahl et al., 2016).

The present findings also demonstrate that instructions targeting retrieval (i.e., the exact phrasing of the MPT instructions at test) exert strong influences on parameters that were conceptualized as measures of processes operating during learning (Hütter et al., 2012). Hence, our results are in line with the notion that there are two qualitatively distinct types of processes operating at retrieval, one memory-dependent and one memory-independent. It is important to note, however, that (a) the MPT parameters have been shown to be differentially sensitive to forgetting (while the $m$-parameter was affected, the $a$-parameter was unaffected; Hütter et al., 2012) and that (b) the $a$-parameter does not increase with a reduction of the quality of encoding (as reflected by a decrease in the $m$-parameter; Mierop et al., in press). These findings are in line with the parameters' conceptualization as indicators of memory-dependent and memoryindependent learning. Nevertheless, our results also show that it is difficult to interpret the MPT parameters as pure indicators of learning processes.

Our results also shed a new light on previous findings. For instance, Hütter and Sweldens (2013) manipulated whether CSs and USs were presented simultaneously or sequentially. In the sequential condition, the US onset occurred only after the CS was erased from the screen. Hütter 
and Sweldens (2013) showed that memory-independent learning arose with simultaneous pairings only. They concluded that the concurrent experience of affect is crucial for memoryindependent learning and interpreted this as evidence for the idea that association formation is more likely to arise as the result of simultaneous than sequential pairings. Because the present findings suggest that mere propositional knowledge is sufficient to produce a significant $a$ parameter, one could wonder why sequential pairings did not bring about a significant $a$ parameter even though this condition also should have led to propositional knowledge about the pairings. One answer might be that response thresholds also had an impact on the findings by Hütter and Sweldens (2013). For instance, in the light of the present findings, it is conceivable that participants focused more strongly on memory in the sequential condition which could have had an adverse effect on estimates of the $a$-parameter. Because we also found that feeling instructions at retrieval can counteract the adverse effect of a focus on memory, it would be interesting to see if the results of Hütter and Sweldens (2013) would change if an emphasis on feelings would be added to their procedure.

\section{Future Directions}

As we noted earlier, uncertainty remains about the way in which instructions about CSUS pairings lead to changes in liking. First, one could conceptualize the instructed procedure as a single pairing of the CS with a US (i.e., a valent adjective) in close spatio-temporal proximity

(Gawronski \& Bodenhausen, 2006). On the basis of this idea, instructions could have an effect on liking, because they result in the formation of associations. Second, instructions might be conceptualized as symbolic events that convey the relation between CS and US (De Houwer \& Hughes, 2016). This implies that the effects of instructions on liking are determined not by the co-occurrence of stimuli within those instructions, but by the symbolic meaning that the instructions as a whole convey. More studies are needed to disentangle these two options (see De 
Houwer \& Hughes, 2016, for suggestions). Our studies indicate that it would be interesting to use MPT procedures also in these future studies.

One difference that currently precludes clear inferences with regard to the nature of learning in the instructed and the experienced procedures concerns the number of different USs used in the present research. While we used eight different US pictures per CS in the experienced procedure, we used only a single attribute ("pleasant" or "unpleasant”) as the US in the instructed procedure. Sweldens and colleagues (2010) argued that multiple USs foster the encoding of a direct link between the CS and the unconditioned reaction (UR). These CS-UR representations have been shown to be more robust towards post-learning modifications of memory (i.e., retroactive interference, US revaluation) than CS-US associations that were constructed when a CS was paired repeatedly with the same US. Future extensions of this research should include manipulations of single US or multiple USs per CS to investigate whether this factor influences the estimate of the $a$-parameter, and therewith, conclusions regarding cognitive processes operating in instructed versus experienced procedures.

The present findings demonstrate that the size of the $a$-parameter can depend on a focus on feelings at retrieval. As all conditions with feelings instructions also included memory instructions, we can, however, not determine whether one would obtain the same effects of feelings instructions without memorization instructions. EC paradigms usually entail a spontaneous focus on evaluative responses given that most EC procedures are framed as impression formation phases (as in the pairing condition in Experiment 1) and contain evaluative pre-ratings of the CSs (Hofmann et al, 2010). Hence, this question is relevant for the generalizability of the present findings to more conventional EC procedures.

Finally, the present research suggests that the $m$ - and $a$-parameters draw on separate strength functions. This notion is in line with a hybrid model that combines the dual-process 
account with the signal detection framework (Wixted, 2007) by assuming that there are two separate strength functions for memory signals and evaluative signals, respectively, along which participants place separate response thresholds. If this is indeed the case, it should be possible to manipulate the respective response thresholds separately. So far, we found them both influenced (i.e., increased) by a focus on feelings, which might not be surprising as both parameters reflect (memory for) affective content. Selective manipulations of memory thresholds, for instance, might be achieved by the use of distractors of varying similarity during the retrieval task (Bayen, Murnane, \& Erdfelder, 1996).

\section{Conclusion}

We investigated whether instructions have the potential to generate memory-independent evaluative learning as indexed by an MPT model of memory-dependency (Hütter et al., 2012). Moreover, the present research addressed whether the indicators of memory-dependent and memory-independent learning are indeed qualitatively different. Using the MPT model, we show a significant contribution of memory-independent learning even when participants are merely instructed about the pairings, but never experience them. These findings raise caution towards the interpretation of the memory-independent MPT parameter as an indicator of association formation, while stressing the functional independence of memory-dependent and memoryindependent indicators of EC effects. Moreover, this research demonstrates the need to clearly distinguish between processes operating at encoding and processes operating at retrieval in empirical and theoretical approaches to EC. 


\section{References}

Batchelder, W. H., \& Riefer, D. M. (1999). Theoretical and empirical review of multinomial process tree modeling. Psychonomic Bulletin \& Review, 6, 57-86.

Bayen, U. J., Murnane, K., \& Erdfelder, E. (1996). Source discrimination, item detection, and multinomial models of source monitoring. Journal of Experimental Psychology: Learning, Memory, and Cognition, 22, 197-215.

Dedonder, J., Corneille, O., Bertinchamps, D., \& Yzerbyt, V. (2013). Overcoming correlational pitfalls: Experimental evidence suggests that evaluative conditioning occurs for explicit but not implicit encoding of CS-US pairings. Social Psychological and Personality Science, 5, 250-257.

De Houwer, J. (2003). A structural analysis of indirect measures of attitudes. In J. Musch \& K.C. Klauer (Eds.), The Psychology of Evaluation: Affective Processes in Cognition and Emotion (pp. 219-244). Mahwah, NJ: Lawrence Erlbaum.

De Houwer, J. (2006). Using the implicit association test does not rule out an impact of conscious propositional knowledge on evaluative conditioning. Learning and Motivation, 37, 176-187.

De Houwer, J. (2014). A Propositional Model of Implicit Evaluation. Social and Personality Psychology Compass, 8, 342-353.

De Houwer, J., \& Hughes, S. (2016). Evaluative conditioning as a symbolic phenomenon: On the relation between evaluative conditioning, evaluative conditioning via instructions, and persuasion. Social Cognition, 34, 480-494.

Gast, A. \& De Houwer, J. (2012). Evaluative conditioning without directly experienced pairings of the conditioned and the unconditioned stimuli. The Quarterly Journal of Experimental Psychology, 65, 1657-1674. 
Gast, A., \& Rothermund, K. (2011). What you see is what will change: Evaluative conditioning effects depend on a focus on valence. Cognition and Emotion, 25, 89-110.

Gawronski, B., \& Bodenhausen, G. V. (2006). Associative and propositional processes in evaluation: An integrative review of implicit and explicit attitude change. Psychological Bulletin, 132, 692-731.

Gawronski, B., \& Walther, E. (2012). What do memory data tell us about the role of contingency awareness in evaluative conditioning? Journal of Experimental Social Psychology, 48, 617-623.

Hofmann, W., De Houwer, J., Perugini, M., Baeyens, F., \& Crombez, G. (2010). Evaluative conditioning in humans: A meta-analysis. Psychological Bulletin, 136, 390-421.

Hütter, M., \& Klauer, K. C. (2016). Applying processing trees in social psychology. European Review of Social Psychology, 27, 116-159.

Hütter, M., \& Sweldens, S. (2013). Implicit misattribution of evaluative responses: Contingency-unaware evaluative conditioning requires simultaneous stimulus presentations. Journal of Experimental Psychology: General, 142(3), 638-643.

Hütter, M., Sweldens, S., Stahl, C., Unkelbach, C., \& Klauer, K. C. (2012). Dissociating contingency awareness and conditioned attitudes: Evidence of contingency-unaware evaluative conditioning. Journal of Experimental Psychology: General, 141(3), 539-557.

Jones, C. R., Fazio, R. H., \& Olson, M. A. (2009). Implicit misattribution as a mechanism underlying evaluative conditioning. Journal of Personality and Social Psychology, 96(5), 933948.

Jones, C. R., Olson, M. A., \& Fazio, R. H. (2010). Evaluative conditioning: The "how" question. Advances in Experimental Social Psychology, 43, 205-255. 
Klauer, K. C., Stahl, C., \& Voss, A. (2011). Multinomial Models and Diffusion Models. In A. Voss, C. Stahl, \& K. C., Klauer (Eds.), Cognitive methods in social psychology, (pp.331354). La Rioja: Dialnet.

Kim, C., Sweldens, S.T.L.R., \& Hütter, M. (2016). The symmetric nature of affective brand associations: Equal effectiveness of forward versus backward evaluative conditioning. Social Psychological and Personality Science, 7, 61-68.

Mitchell, C. J., De Houwer, J., \& Lovibond, P. F. (2009). The propositional nature of human associative learning. Behavioral and Brain Sciences, 32, 183-198.

Mierop, A., Hütter, M., \& Corneille, O. (in press). Resource availability and explicit memory largely determine evaluative conditioning effects in a paradigm claimed to be conducive to implicit attitudes acquisition. Social Psychological and Personality Science.

Pleyers, G., Corneille, O., Luminet, O., \& Yzerbyt, V. (2007). Aware and (dis)liking: Item-based analyses reveal that valence acquisition via evaluative conditioning emerges only when there is contingency awareness. Journal of Experimental Psychology: Learning, Memory and Cognition, 33, 130-144.

Reber, A. S. (1993). Implicit learning and tacit knowledge. New York, NY: Oxford University Press.

Rozin, P., \& Millman, L. (1987). Family environment, not heredity, accounts for family resemblances in food preferences and attitudes: A twin study. Appetite, 8, 125-134.

Self, S. G., \& Liang, K.-Y. (1987). Asymptotic properties of maximum likelihood estimators and likelihood ratio tests under nonstandard conditions. Journal of the American Statistical Association, 82, 605-611.

Shanks, D. R. (2009). The associative nature of human associative learning. Behavioral and Brain Sciences, 32, 225-256. 
Shanks, D. R., \& St. John, M. F. (1994). Characteristics of dissociable human learning systems. Behavioral and Brain Sciences, 17, 367-447.

Stahl, C., Haaf, J., \& Corneille, O. (2016). Subliminal evaluative conditioning? Abovechance CS identification may be necessary and insufficient for attitude learning. Journal of Experimental Psychology: General, 145, 1107-1131.

Stahl, C., Unkelbach, C., \& Corneille, O. (2009). On the respective contributions of awareness of unconditioned stimulus valence and unconditioned stimulus identity in attitude formation through evaluative conditioning. Journal of Personality and Social Psychology, 97, 404-420.

Sweldens, S.T.L.R., Corneille, O., \& Yzerbyt, V. (2014). The role of awareness in attitude formation through evaluative conditioning. Personality and Social Psychology Review, 18, 187209.

Sweldens, S.T.L.R., Osselaer, S.M.J. van, \& Janiszewski, C. (2010). Evaluative Conditioning procedures and the resilience of conditioned brand attitudes. Journal of Consumer Research, 37, 473-489.

Swets, J. A. (1996). Signal Detection Theory and ROC Analysis in Psychology and Diagnostics: Collected Papers. Mahwah, NJ: Lawrence Erlbaum Associates.

Wixted, J.T. (2007). Dual-process theory and signal-detection theory of recognition memory. Psychological Review, 114, 152-176.

Zajonc, R, B. (1968). Attitudinal effects of mere exposure. Journal of Personality and Social Psychology, Monograph Supplement, 9, 1-27. 


\section{Footnotes}

${ }^{1}$ Hütter et al. (2012) also implemented a version of the exclusion condition that asked for the reversal of the memory-based responses. Whether participants were asked to reverse their memory-based responses or their evaluations of the CS when they lacked memory for the pairings did not moderate the results.

${ }^{2}$ Note that the null hypothesis of this test, H0: $a=0$, is on the boundary of the parameter space (a being a probability cannot be negative). Therefore, the appropriate reference distribution is an equal mixture of a chi-square distribution with zero degrees of freedom and one with one degree of freedom (Self \& Liang, 1987). The p-values reported are based on this distribution.

${ }^{3}$ In another, previous study, we omitted memorization instructions. In this experiment, we did not obtain an EC effect, despite a moderate $m$-parameter $(m=.47)$. The estimate of the $a$ parameter $(a=.05)$ did not differ significantly from zero. The fact that we did not obtain an EC effect in the first place (unlike to what has been observed several times), however, had us question whether we should interpret the absence of an effect in the $a$-parameter. For that reason, we conducted Experiment 2 that compares a condition with memorization instructions to a control condition that was identical to the study briefly described here. 


\section{Figure Captions}

Figure 1. Processing tree model of the MPT task in the inclusion and exclusion conditions for positively (CS+) and negatively paired CSs (CS-). The rectangles on the left denote the stimuli, the rectangles on the right the responses. The branches of the processing tree represent the combination of cognitive processes postulated by the model. $\mathrm{m}=$ probability of memory for the pairings; $\mathrm{a}$ = probability of acquiring an evaluation in the absence of memory for the pairings; $\mathrm{r}=$ response tendency towards “pleasant”.

Figure 2. Evaluative ratings in Experiment 1. Error bars represent standard errors.

Figure 3. MPT parameter estimates in Experiment 1. Error bars represent 95\% confidence intervals.

Figure 4. Evaluative ratings in Experiment 2. Error bars represent standard errors.

Figure 5. MPT parameter estimates in Experiment 2. Error bars represent 95\% confidence intervals.

Figure 6. Evaluative ratings in Experiment 3. Error bars represent standard errors.

Figure 7. MPT parameter estimates in Experiment 3. Error bars represent 95\% confidence intervals. 

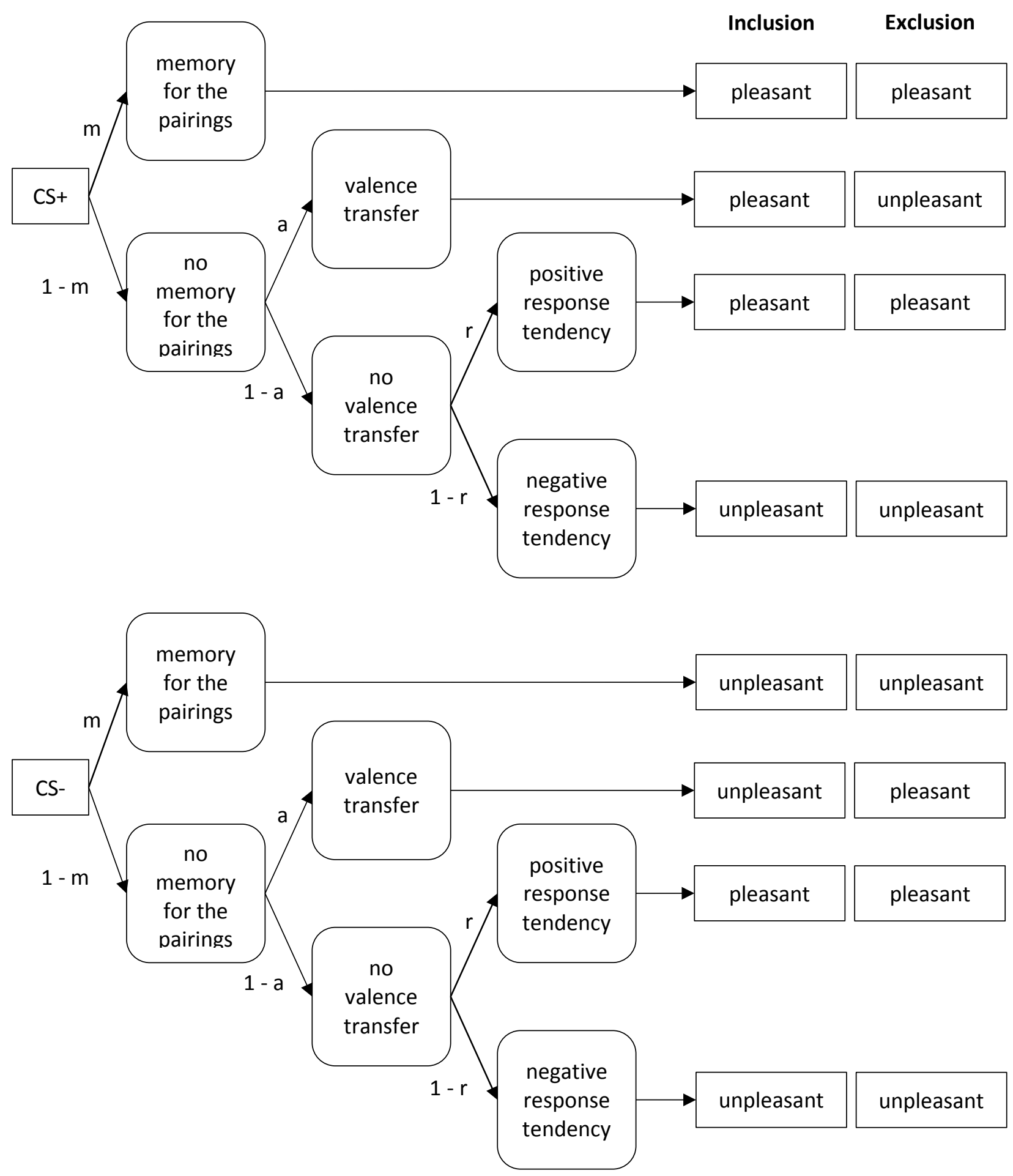

Figure 1. 
115

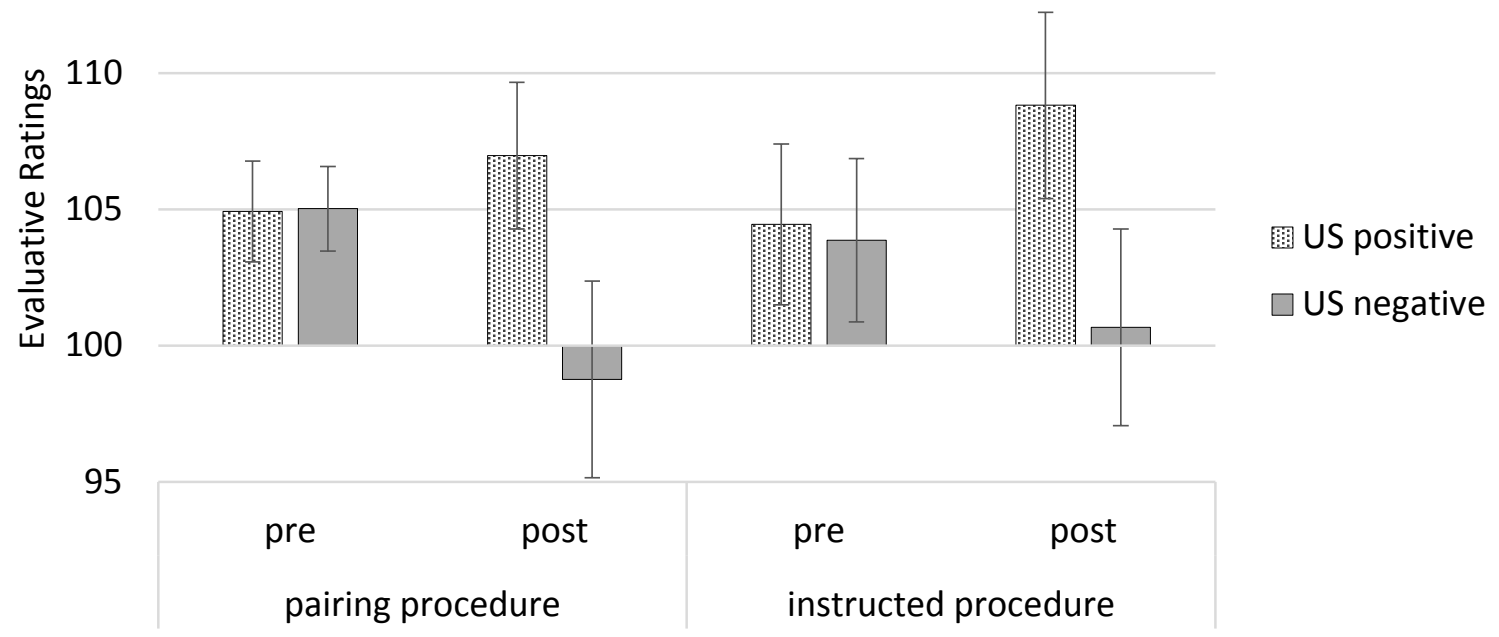

Figure 2. 


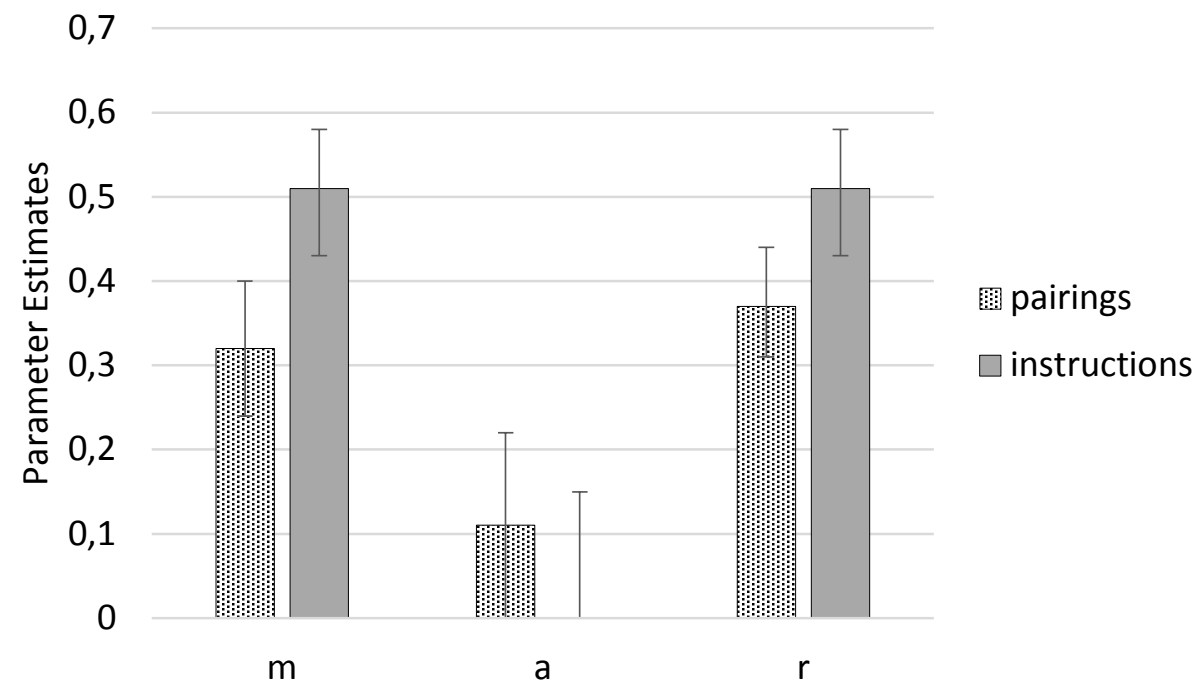

Figure 3. 


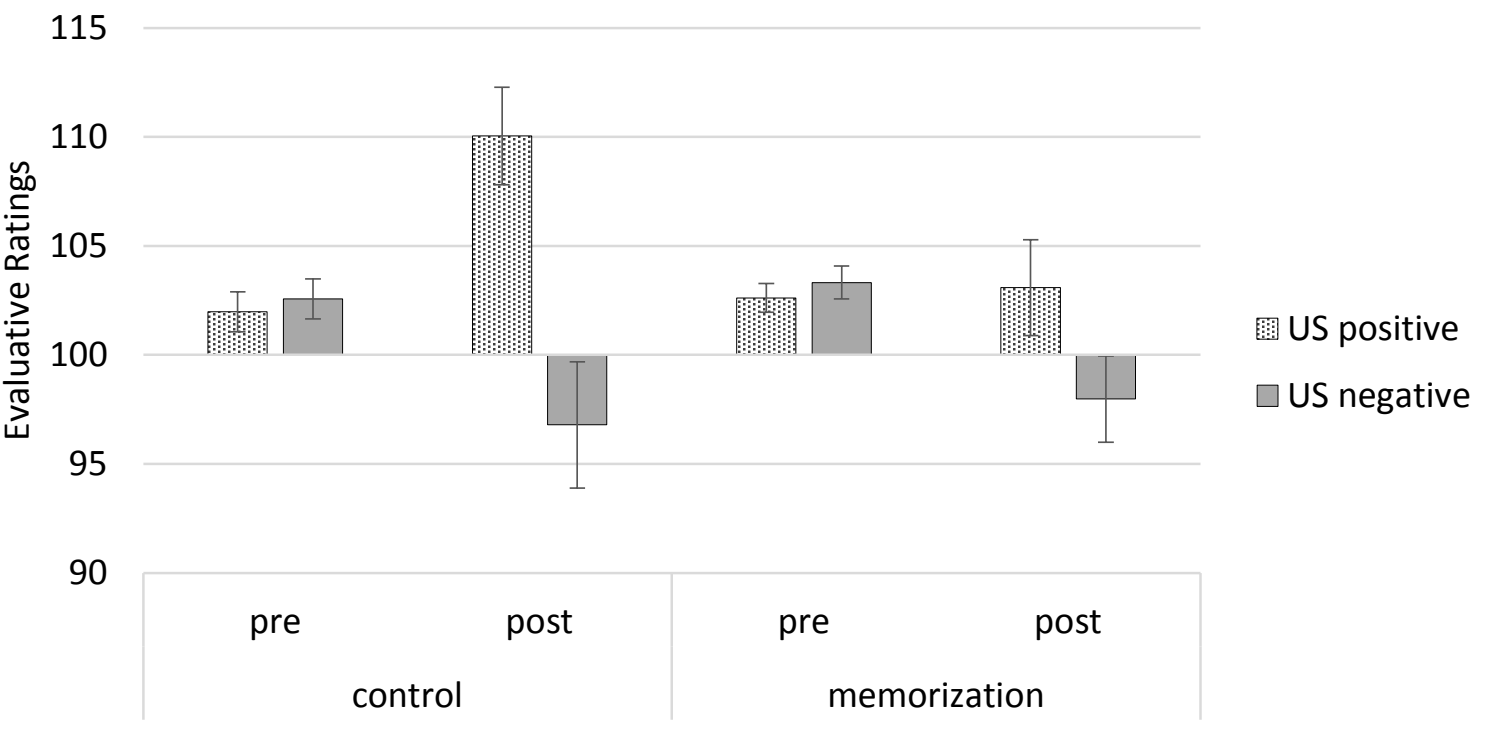

Figure 4. 


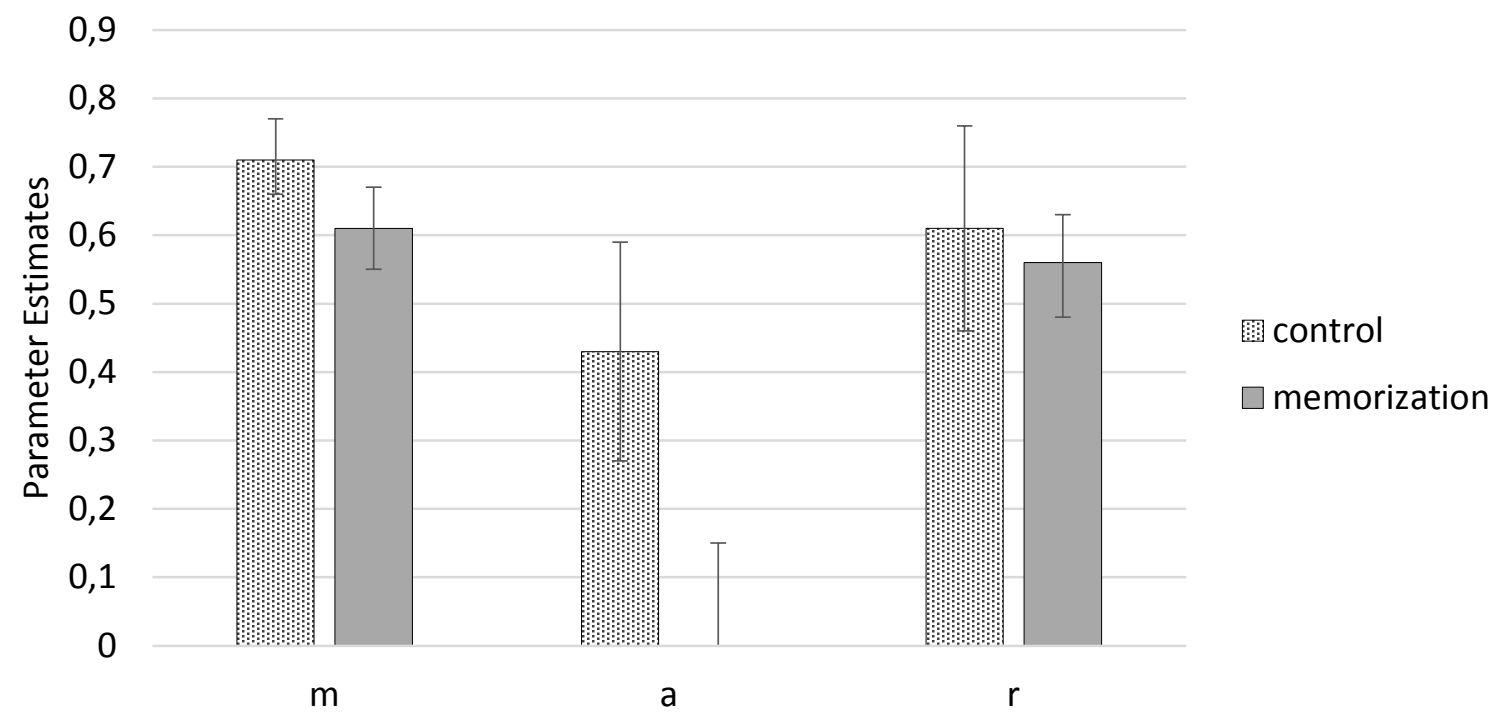

Figure 5. 


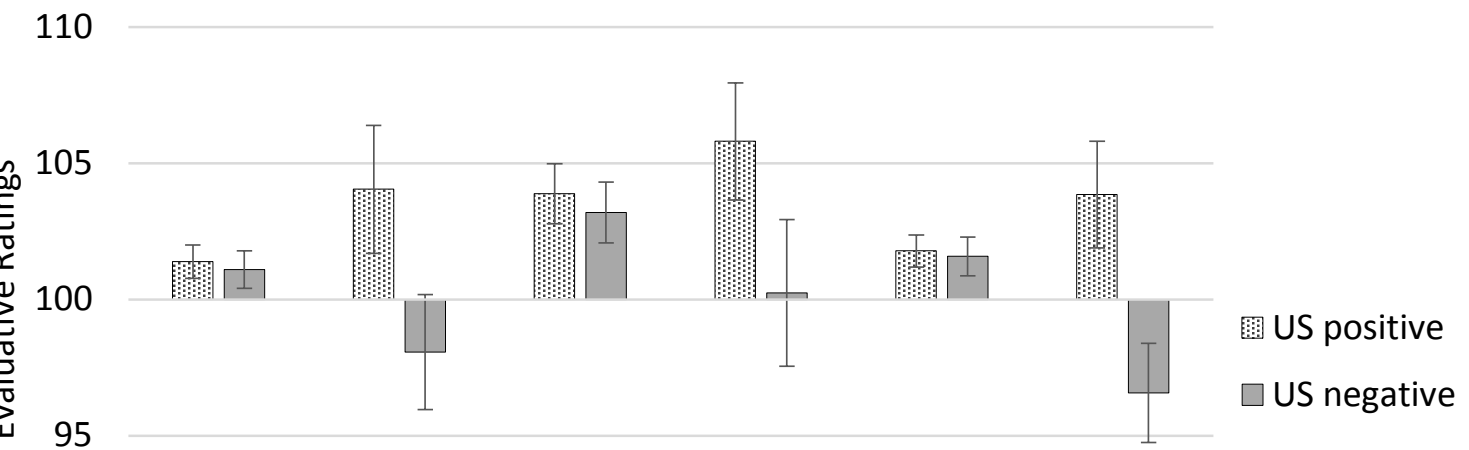

90

\begin{tabular}{|c|c|c|}
\hline $\begin{array}{l}\text { pre post } \\
\text { control }\end{array}$ & $\begin{array}{c}\text { pre post } \\
\text { feelings-at-acquisition }\end{array}$ & $\begin{array}{c}\text { pre post } \\
\text { feelings-at-retrieval }\end{array}$ \\
\hline
\end{tabular}

Figure 6. 


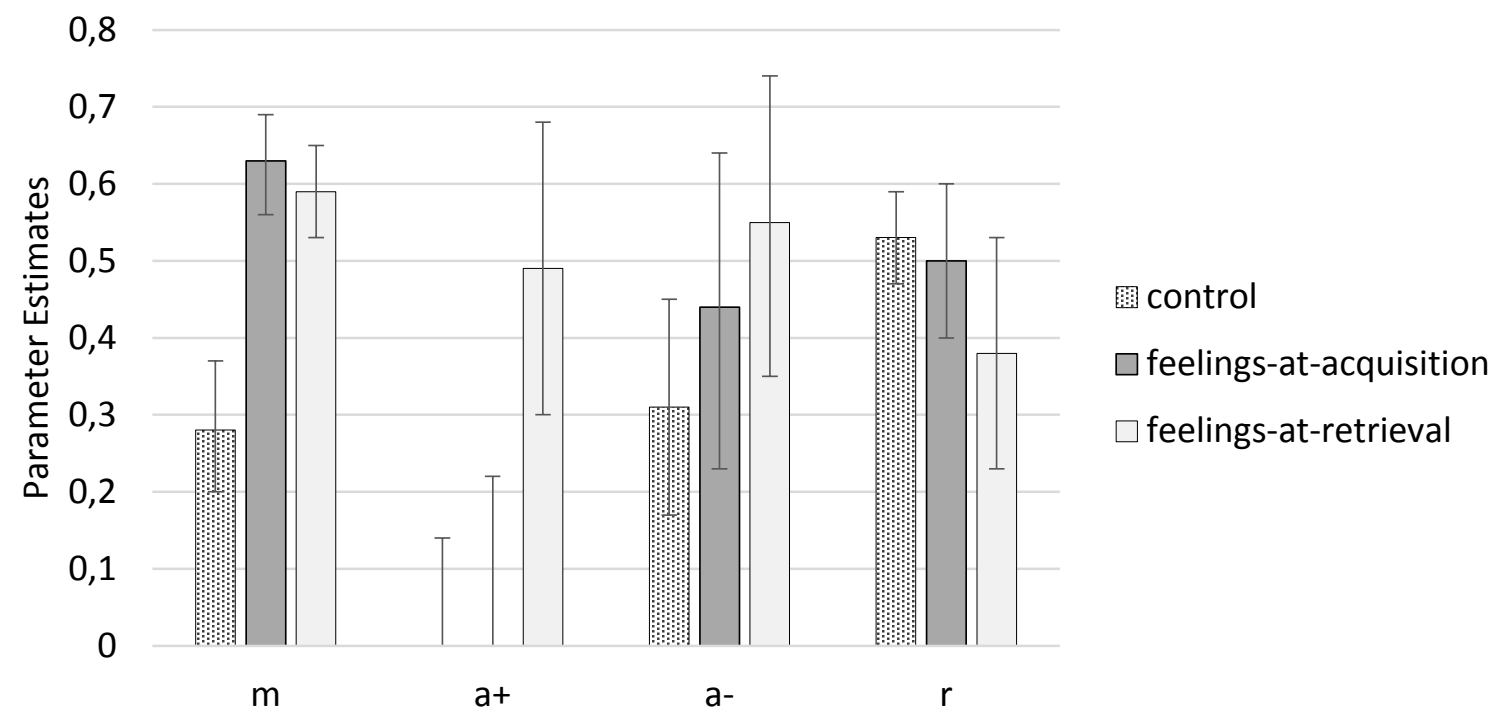

Figure 7. 